\title{
Darstellung und Eigenschaften der Enantiomere des selektiven Antimuscarinikums 1-Cyclohexyl-1-phenyl-4-piperidino-1-butanol (Hexahydro-Difenidol)
}

\author{
Reinhold Tacke $^{+) * a}$, Carsten Strohmann", Stefan Sarge ${ }^{b}$, Heiko K. Cammenga ${ }^{b}$, Dietmar Schomburg , Ernst Mutschler $^{d}$ \\ und Günter Lambrecht ${ }^{d}$
}

Institut für Anorganische und Analytische Chemie der Technischen Universität Braunschweiga Hagenring 30, D-3300 Braunschweig

Institut für Physikalische und Theoretische Chemie der Technischen Universität Braunschweig, Hans-Sommer-Straße 10, D-3300 Braunschweig

GBF (Gesellschaft für Biotechnologische Forschung $\mathrm{mbH}$ ), Mascheroder Weg 1, D-3300 Braunschweig

Pharmakologisches Institut für Naturwissenschaftler der Universität Frankfurt ${ }^{\mathrm{d}}$, Theodor-Stern-Kai 7, Geb. 75A, D-6000 Frankfurt/Main

Eingegangen am 2. September 1988

Keywords: Difenidol, $(R)$ - and (S)-hexahydro- / Antimuscarinic properties / Muscarinic receptor subtypes

Durch Racematspaliung mit (S)-bzw. (R)-Mandeisäure wurden dic Finantiomere von 1-Cyclohexyl-1-phenyt-4-piperidino-2butin-1-ol $[(R)-2$ und $(S)-2]$ dargestellt (Enantiomerenreinheit: $\omega^{2}=99.7 \%$, kalorimetrische Analyse). Katalytische Hydrierung (PdC $(-K o n t a k 1)$ von $(R)-2$ und $(S)-2$ ergab die Enantiomere von 1-Cyclohexyl-1-phenyl-4-piperidino-1-butanol $[\{R\}$ - und (S) Hexahydro-Difenidol, $(R)-1$ and (\$)-1 a]. die als Hydrochloride (R)-1 a $\cdot \mathrm{HCl}$ und $(\mathrm{S})$ - a $\cdot \mathrm{HCl}$ isoliert wurden (ec $=99.7 \%)$. Dic absolute Konliguration der Enantiomere von 1 a und 2 wurde durch Röntgenkristallstrukturanalyse des Manứclats $(S)-1$ a $\cdot(R)$ $\mathrm{C}_{1} \mathrm{H}_{3} \mathrm{CH}(\mathrm{OH}) \mathrm{COOH}$ bestimms. (R)-Hexahydro-Ditenidol $[(R)-$ la] und $(R)-2$ besil\%en eine höhere Affinität zu den atrialen $M 2 x$ und ilealen M2ß-Muscarinrezeptoren des Meerschweinchens als Jie entsprechenden Antipoden (S)-1 a und (S)-2 (atrialer Stereosclcktivitätsindex: $17 \mathrm{bzw}$. 8.6: ilealer Stereoselektivitätsindex: 193 bzw. 44). Darübor hinaus besitzen ( $R$ )-1 a und ( $R$ )-2 eine signifikant hölecte Alínität zu den $M 2 \beta$-Rezeptoren des Jleums als zu den M2x-Rexeptoren des Atriums und sind somil wertrolic Modellverbindungen zur Identifixicrung und Charakterisierung von muscirinischen M2-Subtypen (Atrium/lleum-Quotient: 21 bzw. (0). In Getgensale hiorzu vermögen die schwälher wirksamen (S)Fnimsiomere von 1 a und 2 nicht zwischen den $M \geq x$. und $M 2 \beta$ Rezeptoren zu untersclkeiden.

Kürzlich haben wir über die Darstellung der Racemate der selektiven Antimuscarinika Hexahydro-Difenidol (rac1a) und Hexahydro-Sila-Difenidol ( $r a c-1$ b) berichtet ${ }^{1)}$. Die Silicium-Verbindung rac-1 b ist aufgrund ihres antimuscarinischen Selektivitätsprofils zu einer wichtigen Modellsubstanz geworden, die in der experimentellen Pharmakologie zur Subklassifizierung von Muscarinrezeptoren eingesetzt wird $^{2-17}$. Das Kohlenstoff-Analogon rac-1 a besitzt ein von

*) Neue Anschrift: Prof. Dr. R. Tacke, Institut für Anorganische Chemie der Universität Karlsruhe, Engesserstraße, D-7500 Karlsruhe 1.
Preparation and Propertics of the Enantiomers of the Selective Antimuscaxinic Agent 1-Cyclohexyl-1-phenyl-4-piperidino-Ibutanol (Hexahydro-Difenidol)

Using (S)- or (R)-mandelic acid as the resolving agent, the chantiomers of 1-eyclohexy]-1-phenyl-4-pipcridino-2-butin-1-(s) [(R)-2 and (S)-2] were prepared (enantionseric purity: $e^{2}=49.7^{4}$.u: callsrimetric analysis). Catalytic hydrogenation ( $P d / C$ contaci) of ( $R$ ). 2 and $(S\}-2$ yiclded the conantiomers of 1-cyclohexyl-1-phenyl-tgiperidino-1-butanol $[(R)$ - and (SHhexahydro-dilenidol. $(R)-1$;

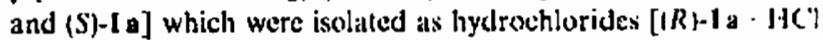

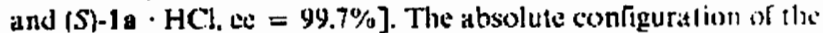
enantiomers of $1 \mathrm{a}$ and 2 was determined by an $\mathrm{X}$-raty erystal structure analysis of the mandelate $(S)-1 \mathrm{a} \cdot(R)-\mathrm{C}_{6} \mathrm{H}_{3}(\mathrm{C})(\mathrm{OH})-$ COOH. (R)-Hexahydro-difcnidol $\left[\left\{R^{\prime}\right)-1 \mathrm{a}\right]$ and $(R)-2$ (xhihil a higher altunity for the atrial $M 2 \alpha$ and ilcal $M 2 \beta$ muscarinic recep. tors of the guinea pig than the respective antipodes (S)-1 a and (S)-2 (alrial stereoselectivity index: 17 and 8.6, respectively: ileal stereoselectivity index: 193 and 44. respetively). In addition. ( $R$. Ia and $(R)-2$ exhibit a signilicantly higher aflinity for the $M 2$, receptors of the iteum than for the $M 2 \alpha$ receptors of the altium (atrium/ileum ratio: 21 and 10 , respectively). Thus. $(K \mid-1$ a and (R)-2 are valuable tools for the identification and characterization of nuscarinic $M 2$ subtypes. In contrast, the less potent i.St-unantiomers of $1 \mathrm{a}$ and $2 \mathrm{do}$ not differentiate between $M 2 x$ and $M 2 \beta$ receptors.

der Silicium-Verbindung abweichendes Selektivitätsprofil und ist ebenfalls sehr gut zur Klassifizierung von Subtypen muscarinischer Rezeptoren geeignet ${ }^{2-6,89}$.

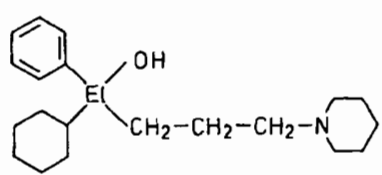

$1 a: E l=C ; \quad 1 b: E l=S i$

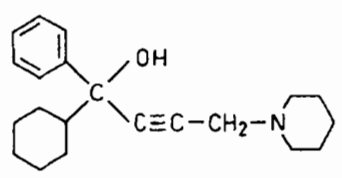

2
Da sowohl die absolute Wirkungsstärke als auch das Selektivitätsprofil von chiralen Antimuscarinika vom Struk- 
turtyp des Hexahydro-Difenidols und Hexahydro-Sila-Difenidols sehr stark von der absoluten Konfiguration abhängen (vgl. Lit. ${ }^{18-21)}$ und dort angegebene Referenzen), war die Verfügbarkeit der Enantiomere des Hexahydro-Difenidols und Hexahydro-Sila-Difenidols als Modellsubstanzen für die experimentelle Pharmakologie wünschenswert: Aus Gründen der Analogie (vgl. Lit. ${ }^{18-21}$ ) war zu erwarten, daß die $(R)$-konfigurierten Enantiomere von $1 \mathrm{a}$ und $\mathbf{1} \mathbf{b}$ den entsprechenden ( $S$ )-Antipoden bezüglich der antimuscarinischen Potenz und Selektivität überlegen sind. Es erschien deshalb lohnenswert, die Enantiomere von Hexahydro-Difenidol und Hexahydro-Sila-Difenidol zu synthetisieren. Da die Darstellung optisch aktiver Silanole in der Regel schwieriger und aufwendiger ist als die Herstellung strukturanaloger Alkohole (vgl. hierzu Lit. ${ }^{21}$ ), haben wir uns zunächst der Synthese der Enantiomere der Kohlenstoff-Verbindung 1 a zugewandt, um dann später mit den hierbei gewonnenen Erfahrungen die Darstellung der Enantiomere des SilaAnalogons $\mathbf{1 b}$ in Angriff zu nehmen. - Wir berichten hier über die Herstellung und pharmakologischen Eigenschaften von $(R)$-Hexahydro-Difenidol $[(R)-1$ a $]$ und $(S)$-HexahydroDifenidol [(S)-1a a , die als Hydrochloride $(R)-1 \mathbf{a} \cdot \mathrm{HCl}$ bzw. $(S)-1 \mathrm{a} \cdot \mathrm{HCl}$ enantiomerenrein gewonnen wurden ${ }^{22)}$. Außerdem werden Darstellung und pharmakologische Eigenschaften der von $(R)-1$ a und $(S)$-1 a abzuleitenden ungesättigten Derivate $(R)-2$ und $(S)-2$ beschrieben.

\section{Synthesen}

Die Synthese der Enantiomere von Hexahydro-Difenidol (1 a) erfolgte durch katalytische Hydrierung (Pd/C-Kontakt) der Enantiomere von 1-Cyclohexyl-1-phenyl-4-piperidino2-butin-1-ol (2), die in enantiomerenreiner Form durch eine Racematspaltung von rac-2 - unter Verwendung von $(S)$ bzw. (R)-Mandelsäure als Hilfsreagenz - zugänglich sind. rac-2 wurde durch Umsetzung von Cyclohexylphenylketon mit 3-Piperidino-1-propin-1-yl-lithium - gefolgt von wässeriger Aufarbeitung - hergestellt ${ }^{23)}$.

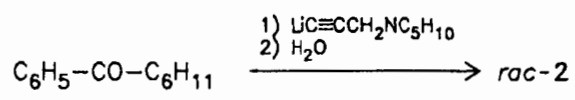

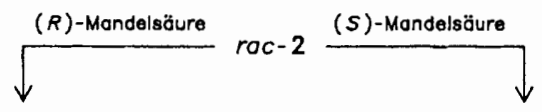

$(S)-2 \cdot(R)-\mathrm{C}_{6} \mathrm{H}_{5} \mathrm{CH}(\mathrm{OH}) \mathrm{COOH}$ $\mathrm{NaOH} \downarrow$

(5) -2

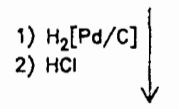

$(S)-1 \mathrm{a} \cdot \mathrm{HCl}$

$(R)-2 \cdot(S)-\mathrm{C}_{6} \mathrm{H}_{5} \mathrm{CH}(\mathrm{OH}) \mathrm{COOH}$ $\downarrow \mathrm{NaOH}$

(R) -2

$$
\text { 1) } \mathrm{H}_{2}[\mathrm{Pd} / \mathrm{Cl}
$$

$(R)-1 \mathrm{a} \cdot \mathrm{HCl}$

Durch Racematspaltung wurden zunächst die gut kristallisierenden Mandelate $(R)-2 \cdot(S)-\mathrm{C}_{6} \mathrm{H}_{5} \mathrm{CH}(\mathrm{OH}) \mathrm{COOH}$ bzw. (S)-2 $\cdot(R)-\mathrm{C}_{6} \mathrm{H}_{5} \mathrm{CH}(\mathrm{OH}) \mathrm{COOH}$ erhalten, die durch dreimaliges fraktionierendes Kristallisieren aus Acetonitril gereinigt wurden. Durch Umsetzung dieser Produkte mit Natronlauge wurden dann die gut kristallisierenden freien Basen $(R)-2$ und $(S)-2$ gewonnen. Deren zweimalige Kristallisation aus Aceton lieferte Präparate mit einer Enantiomerenreinheit von ee $=99.7 \%$. Weiteres Umkristallisieren - sowohl der freien Basen als auch der entsprechenden Mandelate - führte zu keiner weiteren meßbaren Veränderung der physikalischen Eigenschaften (spezifische Drehungen, thermische Eigenschaften). Durch katalytische Hydrierung der so erhaltenen enantiomerenreinen Verbindungen $(R)-2$ und $(S)-2$ wurden dann die jeweiligen Enantiomere von 1 a erhalten, die anschließend - da sie als ölige, nicht kristallisierende Substanzen anfielen - durch Umsetzung mit Chlorwasserstoff in die entsprechenden Hydrochloride $(R)-\mathbf{1}$ a $\cdot \mathrm{HCl}$ und $(S)-1 \mathbf{a} \cdot \mathrm{HCl}$ übergeführt und als solche durch zweimaliges Kristallisieren aus 2-Propanol/ Diethylether abschließend gereinigt wurden. Für die so erhaltenen Präparate wird auf die gleiche Enantiomerenreinheit geschlossen, wie sie für die Vorstufen $(R)-2$ und $(S)$ 2 experimentell bestimmt wurde $(\mathrm{ee}=99.7 \%)$.

Die absolute Konfiguration der Enantiomere von 1a (bzw. 1a $\cdot \mathrm{HCl}$ ) und 2 [bzw. $\left.2 \cdot \mathrm{C}_{6} \mathrm{H}_{5} \mathrm{CH}(\mathrm{OH}) \mathrm{COOH}\right]$ wurde durch Röntgenstrukturanalyse des Mandelats (S)-1a . $(R)-\mathrm{C}_{6} \mathrm{H}_{5} \mathrm{CH}(\mathrm{OH}) \mathrm{COOH}$ ermittelt, das durch Umsetzung von (S)-1 a mit $(R)$-Mandelsäure dargestellt wurde.

Die Enantiomerenreinheit von $(R)-2$ und $(S)$-2 wurde kalorimetrisch bestimmt. Die mit einem dynamischen Wärmestrom-Differenz-Kalorimeter ermittelten Schmelztemperaturen $\left(\vartheta_{\text {fus }}\right)$, Schmelzenthalpien $\left(\Delta_{\text {fus }} H^{\ominus}\right)$ und eutektischen Reinheiten $(X)$ sind in Tab. 1 aufgeführt. Danach ergibt sich für $(R)-2$ und (S)-2 eine Enantiomerenreinheit von

Tab. 1. Kalorimetrisch bestimmte Daten von rac-2, $(R)-2$ und (S)-2

\begin{tabular}{cccc}
\hline Verbindung & $\vartheta_{\text {fus }} /{ }^{\circ} \mathrm{C}$ & $\begin{array}{c}\Delta_{\text {fus }} H^{\ominus} / \mathrm{kJ} \\
\mathrm{mol}^{-1}\end{array}$ & $X / \mathrm{mol}-\%$ \\
\hline rac-2 & 134.4 & 40.2 & \\
$(R)-2$ & 126.5 & 35.6 & 99.7 \\
$(S)-2$ & 126.5 & 36.0 & 99.7 \\
\hline
\end{tabular}

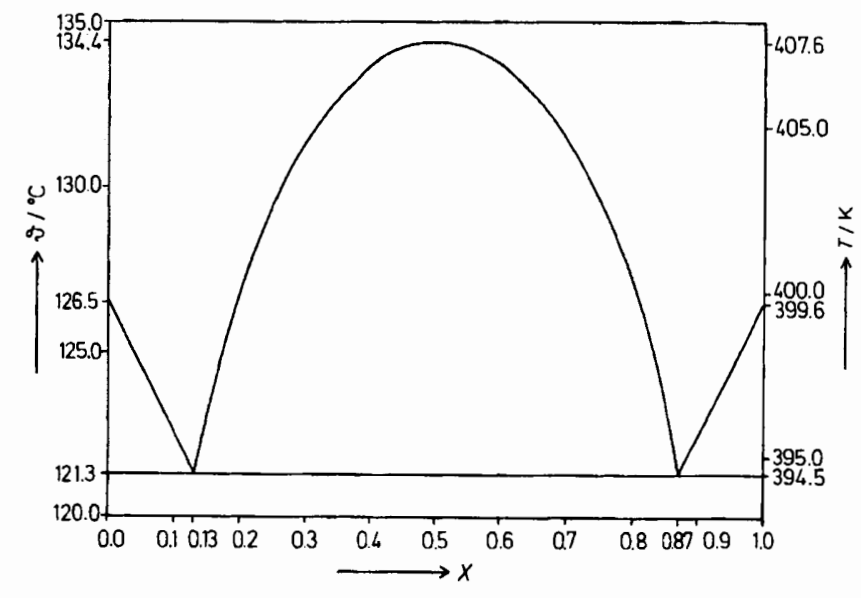

Abb. 1. Phasendiagramm des Systems (R)-2/(S)-2 
ee $=99.7 \%$. Die beiden Enantiomere bilden ein dystektisches Phasensystem; Abb. 1 zeigt das mit den Daten aus Tab. 1 berechnete Phasendiagramm.

Da man davon ausgehen kann, daß die katalytische Hydrierung von $(R)-2$ und $(S)-2$ keine chemischen Veränderungen am Chiralitätszentrum beinhaltet und deshalb auch keine Veränderung der Enantiomerenreinheit mit sich bringt, wird für $(R)-\mathbf{1} \mathbf{a} \cdot \mathrm{HCl}$ und $(S)-\mathbf{1} \mathbf{a} \cdot \mathrm{HCl}$ auf die gleiche Enantiomerenreinheit geschlossen, wie sie für die Enantiomere von 2 kalorimetrisch bestimmt wurde (ee $=99.7 \%$; eine direkte kalorimetrische Reinheitsbestimmung der Enantiomere von $1 \mathrm{a} \cdot \mathrm{HCl}$ war nicht möglich, da sich die Substanzen beim Erhitzen zersetzten; Versuche zur ${ }^{1} \mathrm{H}$ - und ${ }^{13} \mathrm{C}$-NMR-spektroskopischen Reinheitsbestimmung auf der Stufe der freien Basen $(R)-\mathbf{1}$ a und $(S)-1 \mathbf{a}$ in Gegenwart optisch aktiver Verschiebungsreagenzien - wie z. B. $\mathrm{Eu}(\mathrm{hfc})_{3}$ - blieben ebenfalls erfolglos.

Die absoluten Konfigurationen der Enantiomere von $1 \mathbf{a}$, 2, $1 \mathrm{a} \cdot \mathrm{HCl}$ und $2 \cdot \mathrm{C}_{6} \mathrm{H}_{5} \mathrm{CH}(\mathrm{OH}) \mathrm{COOH}$ wurden auf der Grundlage der Ergebnisse der Röntgenstrukturanalyse des aus 2-Propanol/Diethylether (1:2) kristallisierenden Mandelats $(S)-\mathbf{1} \mathbf{a} \cdot(R)-\mathrm{C}_{6} \mathrm{H}_{5} \mathrm{CH}(\mathrm{OH}) \mathrm{COOH}$ bestimmt. Letzteres wurde aus dem in Chloroform-Lösung linksdrehenden Hexahydro-Difenidol-hydrochlorid $[(S)-1 \mathbf{a} \cdot \mathrm{HCl}]$ hergestellt, indem man die entsprechende Base $(S)$-1 a freisetzte und diese dann mit $(R)$-Mandelsäure umsetzte. Die Ergebnisse der Röntgenstrukturanalyse sind in Abb. 2 sowie in Tab. 3 und Tab. 4 zusammengefaßt.

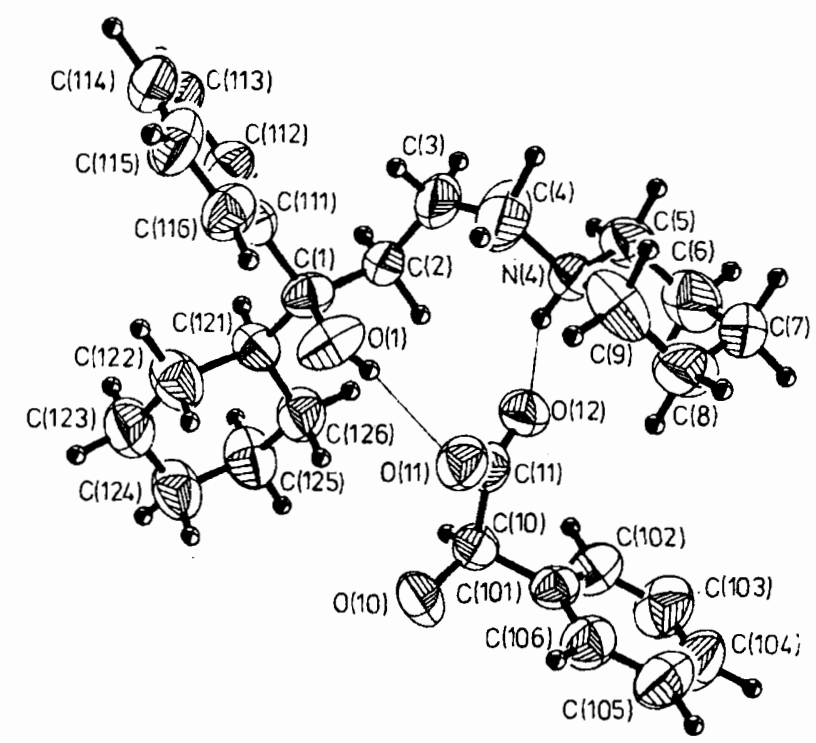

Abb. 2. Perspektivische Darstellung der Ionenpaar-Struktur von $(S)-1 \mathbf{a} \cdot(R)-\mathrm{C}_{6} \mathrm{H}_{5} \mathrm{CH}(\mathrm{OH}) \mathrm{COOH}$ mit Angabe des Numerierungssystems

Die Bindungsparameter von $(S)-\mathbf{1 a} \cdot(R)-\mathrm{C}_{6} \mathrm{H}_{5} \mathrm{CH}(\mathrm{OH})-$ $\mathrm{COOH}$ liegen mit Ausnahme des extrem kurzen Abstands zwischen $C(3)$ und $C(4)$ (127 pm) in den üblicherweise gefundenen Bereichen. Die stark anisotropen Temperaturfaktoren (vgl. Abb. 2) weisen darauf hin, daB hier wahrscheinlich eine Fehlordnung vorliegt, die zu einer scheinbaren Bindungsverkürzung führt.
Im Kristallverband bilden Kation und Anion durch zwei Wasserstoffbrücken stabilisierte Ionenpaare. Diese werden zwischen dem $\mathrm{O}(1)$-Atom der OH-Gruppe des Kations und dem $\mathrm{O}(11)$-Atom der Carboxylat-Gruppe des Anions $[\mathrm{O}(1) \cdots \mathrm{O}(11) 285 \mathrm{pm}]$ sowie zwischen dem N(4)-Atom der Ammonium-Gruppe des Kations und dem O(12)-Atom der Carboxylat-Gruppe des Anions [N(4) $\cdots \mathrm{O}(12) 270.6 \mathrm{pm}$ ] ausgebildet. Die vom Kation angenommene Konformation der zentralen Propylen-Gruppe dürfte weitgehend durch die aufgrund der Wasserstoffbrücken vorgegebenen geometrischen Randbedingungen bestimmt sein [Torsionswinkel: $\mathrm{C}(1)-\mathrm{C}(2)-\mathrm{C}(3)-\mathrm{C}(4) \quad 92.7^{\circ}, \quad \mathrm{C}(2)-\mathrm{C}(3)-\mathrm{C}(4)-\mathrm{N}(4)$ $43.8^{\circ} \mathrm{]}$.

Die absolute Konfiguration des Kations [(S)-Konfiguration] ergibt sich aus der bekannten Konfiguration des Anions der eingesetzten (R)-Mandelsäure. Auch die Röntgenstrukturanalyse selbst ergibt aufgrund von drei voneinander unabhängigen Tests $\left(R_{\mathrm{G}^{-}}\right.$-Wert-Vergleich, Analyse der „empfindlichsten“" Reflexe und Rogers-Test ${ }^{24}$ ) die „richtige“ absolute Konfiguration, wenngleich auch mit nicht ausreichender Signifikanz.

Da bei den beschriebenen chemischen Umwandlungen die Konfigurations-Erhaltung der zentralen Kohlenstoffatome vorausgesetzt werden kann, ergeben sich aus den Ergebnissen dieser Röntgenstrukturanalyse auch die absoluten Konfigurationen aller übrigen dargestellten Verbindungen.

\section{Pharmakologische Eigenschaften}

Die Enantiomere von Hexahydro-Difenidol (1a) und 2 wurden an den Muscarinrezeptoren des elektrisch gereizten linken Atriums und der isolierten Longitudinalmuskulatur des Ileums des Meerschweinchens hinsichtlich ihrer antimuscarinischen Aktivität untersucht, wobei Arecaidinpropargylester ${ }^{2)}$ als Agonist diente.

In den letzten Jahren konnte mit Hilfe selektiver Antagonisten gezeigt werden, daß diese kardialen und ilealen Muscarinrezeptoren keine einheitliche Population darstellen, sondern als Subtypen klassifiziert werden können ${ }^{2-6,10-12,19-21,25,26)}$. Wir haben vorgeschlagen ${ }^{3-6)}$, die atrialen Muscarinrezeptoren als $M 2 \alpha$ - und die ilealen als M2 $\beta$-Rezeptoren zu bezeichnen.

Die Ergebnisse der pharmakologischen Untersuchungen mit den Enantiomeren von 1 a (durchgeführt mit den entsprechenden Hydrochloriden $\mathbf{1 a} \cdot \mathrm{HCl}$ ) und mit den Enantiomeren von 2 an den $\mathrm{M} 2 \alpha$ - und $\mathrm{M} 2 \beta$-Rezeptoren sind in Tab. 2 und Abb. 3 zusammengestellt.

Die Enantiomere von 1 a und 2 verhalten sich an den postsynaptischen M2 $\alpha$-Rezeptoren des Atriums und an den M2 $\beta$-Rezeptoren des lleums als Antagonisten gegenüber dem selektiven muscarinischen Agonisten Arecaidinpropargylester.

Alle Verbindungen erfüllen die üblichen Kriterien ${ }^{27-299}$ für einen rein kompetitiven Antagonismus. So sind z. B. die in Tab. 2 angeführten Steigungen in den Arunlakshana-Schild-Darstellungen von dem für einen kompetitiven Antagonismus theoretisch zu fordernden Wert von -1.00 nicht signifikant verschieden $(p>0.05)$.

Wie die Daten in Tab. 2 und Abb. 3 zeigen, sind die $(R)$ konfigurierten Enantiomere von 1 a und 2 sowohl am 
Tab. 2. Pharmakologische Parameter für den Antagonismus zwischen Arecaidinpropargylester und den Enantiomeren von 1 a und 2 an den muscarinischen M2 $\alpha$-Rezeptoren des elektrisch gereizten linken Atriums und an M2 $\beta$-Rezeptoren der Longitudinalmuskulatur des lleums vom Meerschweinchen

\begin{tabular}{|c|c|c|c|c|c|c|c|c|c|}
\hline \multirow{2}{*}{$\begin{array}{l}\text { Verbin- } \\
\text { dung }\end{array}$} & \multicolumn{4}{|c|}{ Atrium } & \multicolumn{4}{|c|}{ Ileum } & \multirow{2}{*}{$A I-Q^{\mathrm{d})}$} \\
\hline & $\mathrm{p} A_{2}-$ Wert $^{\mathrm{a})}$ & $\begin{array}{c}K_{\mathrm{D}}-\text { Wert } \\
{[\mathrm{mol} / \mathrm{l}]}\end{array}$ & Steigung ${ }^{\text {b) }}$ & $S I^{\mathrm{c})}$ & $\mathrm{p} A_{2}-$ Wert $^{\mathrm{a})}$ & $\begin{array}{c}K_{\mathrm{D}}-\text { Wert } \\
{[\mathrm{mol} / \mathrm{l}]}\end{array}$ & Steigung ${ }^{\text {b) }}$ & $S I^{\mathrm{c})}$ & \\
\hline $\begin{array}{l}(R)-1 \mathrm{a} \\
(S)-1 \mathrm{a} \\
(R)-2 \\
(S)-2\end{array}$ & $\begin{array}{l}7.03 \pm 0.06 \\
5.80 \pm 0.07 \\
7.77 \pm 0.04 \\
6.84 \pm 0.05\end{array}$ & $\begin{array}{l}9.41 \times 10^{-8} \\
1.59 \times 10^{-6} \\
1.69 \times 10^{-8} \\
1.45 \times 10^{-7}\end{array}$ & $\begin{array}{l}-0.83 \pm 0.30 \\
-0.96 \pm 0.19 \\
-1.09 \pm 0.07 \\
-1.03 \pm 0.13\end{array}$ & 8.6 & $\begin{array}{l}8.35 \pm 0.04 \\
6.07 \pm 0.05 \\
8.78 \pm 0.04 \\
7.14 \pm 0.04\end{array}$ & $\begin{array}{l}4.44 \times 10^{-9} \\
8.56 \times 10^{-7} \\
1.66 \times 10^{-9} \\
7.24 \times 10^{-8}\end{array}$ & $\begin{array}{l}-0.92 \pm 0.07 \\
-0.87 \pm 0.17 \\
-1.10 \pm 0.06 \\
-0.99 \pm 0.07\end{array}$ & 193 & $\begin{array}{l}21 \\
1.9 \\
10 \\
2.0\end{array}$ \\
\hline
\end{tabular}

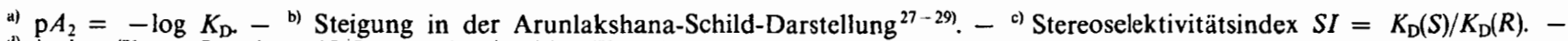
d) Atrium/Ilcum-Quotient $A I-Q=K_{\mathrm{D}}($ Atrium $) / K_{\mathrm{D}}$ (Ileum).

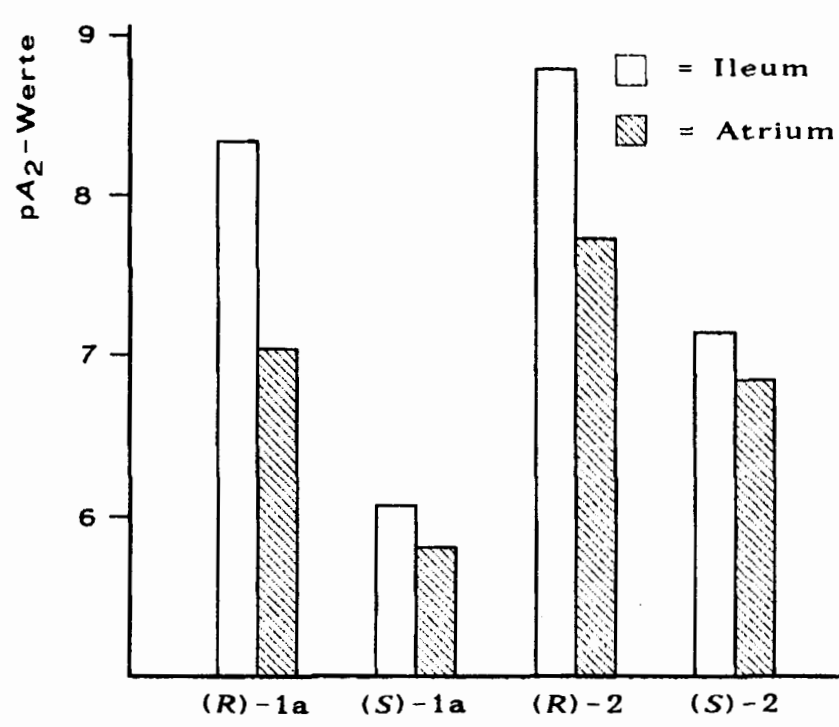

Abb. 3. Affinitäten ( $\mathrm{p} A_{2}$-Werte) der Enantiomere von 1 a und 2 zu den postsynaptischen Muscarinrezeptoren der Atriummuskulatur

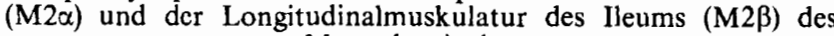
Meerschweinchens

Atrium als auch am Ileum wesentlich stärker antimuscarinisch wirksam als die entsprechenden (S)-Antipoden. Wie sich den Stereoselektivitätsindices von 17 bzw. 193 für 1 a und 8.6 bzw. 44 für 2 entnehmen läßt, besitzt 1 a sowohl an den M2 $\alpha$ - als auch an den M2 $\beta$-Rezeptoren die größere Stereoselektivität. Bemerkenswert ist weiterhin, da $\beta$ die beiden Muscarinrezeptor-Subtypen quantitativ unterschiedliche Anforderungen an die Geometrie der Enantiomere von 1 a und 2 stellen: Die ilealen M2 $\beta$-Rezeptoren zeigen deutlich höhere Stereoselektivitätsindices als die atrialen $M 2 \alpha$ Rezeptoren. Vergleichbare Befunde erhielten wir bei der pharmakologischen Prüfung der Enantiomere des Procyclidins und Tricyclamol-iodids ${ }^{19,20)}$. Dies bedeutet, daß man bei Vertretern dieses Strukturtyps neben den Unterschieden in den absoluten Affinitäten auch die unterschiedlichen Stereoselektivitätsindices an Atrium und Ileum als weiteres Kriterium bei der Rezeptordifferenzierung einsetzen kann.

Mit p $A_{2}$-Werten von 8.35 und 8.78 besitzen $(R)$-Hexahydro-Difenidol $[(R)-1 \mathrm{a}]$ und $(R)-2$ eine signifikant höhere Affinität zu den M2 $\beta$-Rezeptoren des Ileums im Vergleich $\mathrm{zu}$ den atrialen $\mathrm{M} 2 \alpha$-Rezeptoren $\left(\mathrm{p} A_{2}=7.03\right.$ bzw. 7.77;
Atrium/Ileum-Quotient $=21$ bzw. 10). $(R)-1 \mathrm{a}$ und $(R)-2$ sind somit weitere wichtige Modellverbindungen zur Identifizierung und Charakterisierung von muscarinischen M2Subtypen. Die schwächer antimuscarinisch wirksamen (S)Enantiomere von 1 a und 2 vermögen dagegen nicht zwischen den $\mathrm{M} 2 \alpha$ - und M2 $\beta$-Rezeptoren zu unterscheiden; die entsprechenden $\mathrm{p} A_{2}$-Werte (Tab. 2) an Atrium und Ileum sind nicht signifikant verschieden $(p>0.05)$.

Die Dehydrierung der Enantiomere von Hexahydro-Difenidol (1 a) zu den entsprechenden Alkin-Derivaten 2 führt an beiden Organen zu einer Zunahme der Affinität zu den Muscarinrezeptoren um bis zu einer Zehnerpotenz. Diese Affinitätszunahme ist jedoch mit einer Verringerung der Stereoselektivität und im Falle von $(R)-2$ außerdem mit einer etwas geringeren Rezeptor-Selektivität verbunden.

R. T. dankt der Deutschen Forschungsgemeinschaft und dem Fonds der Chemischen Industrie für die finanzielle Unterstützung dieser Arbeit und der Bayer $A G$, Leverkusen und Wuppertal-Elberfeld, für großzügige Chemikalienspenden. C. St. dankt dem Land Niedersachsen für ein Promotionsstipendium (Graduierten-Förderungsgesetz).

\section{Experimenteller Teil}

a) Synthesen

Schmelzpunkte (unkorrigiert): Kofler-Heiztischmikroskop (Fa. Reichert). - Optische Drehungen: Polarimeter 241 (Fa. PerkinElmer). Das für die Messungen verwendete Chloroform (LiChrosolv ${ }^{\mathfrak{x}}$, Merck) wurde unmittelbar vor Gebrauch säulenchromatographisch mit $\mathrm{Al}_{2} \mathrm{O}_{3}$ (Akt. I) getrocknet und gereinigt (40 $\mathrm{g} \mathrm{Al}_{2} \mathrm{O}_{3} / 100 \mathrm{ml} \mathrm{CHCl}$ ). - ${ }^{\mathrm{t}} \mathrm{H}$-NMR-Spektren [400.1 $\mathrm{MHz}$, Lösungsmittel $\mathrm{CDCl}_{3}$, int. Standard TMS $\left.(\delta=0)\right]$ : AM-400-Gerät (Fa. Bruker). - ${ }^{13} \mathrm{C}-\mathrm{NMR}$-Spektren $[100.6 \mathrm{MHz}$, Lösungsmittel und int. Standard $\left.\mathrm{CDCl}_{3}(\delta=77.05)\right]$. Die Zuordnung der ${ }^{13} \mathrm{C}$ NMR-Signale wurden durch DEPT-Experimente unterstützt. FAB-Massenspektren (Matrix: Glycerin; Neutralgas: Xenon): Gerät 8430 (Fa. Finnigan-MAT).

( \pm )-1-Cyclohexyl-1-phenyl-4-piperidino-2-hutin-1-ol (rac-2): $\mathrm{Zu}$ einer Lösung von $33.9 \mathrm{~g}(0.18 \mathrm{~mol})$ Cyclohexylphenylketon in $300 \mathrm{ml}$ Diethylether wurde bei $0^{\circ} \mathrm{C}$ unter Rühren innerhalb von $30 \mathrm{~min}$ eine Lösung von 3-Piperidino-1-propin-1-yl-lithium in Hexan/Diethylether getropft, die zuvor durch Umsetzung einer Lösung von $22.2 \mathrm{~g}(0.18 \mathrm{~mol}) 3$-Piperidino-1-propin in $150 \mathrm{ml}$ Diethylether mit $113 \mathrm{ml}$ einer $1.6 \mathrm{M} n$-Butyllithium-Lösung in Hexan 
( $\triangleq 0.18 \mathrm{~mol} n \mathrm{BuLi}$ ) bei $-50^{\circ} \mathrm{C}$ hergestellt wurde. Anschließend rührte man $16 \mathrm{~h}$ bei Raumtemp., versetzte dann vorsichtig mit $150 \mathrm{ml}$ Eis/Wasser, trennte die organische Phase ab, extrahierte die wässerige Lösung mit Diethylether, wusch die vereinigten etherischen Extrakte mit wenig Wasser und trocknete mit wasserfreiem $\mathrm{Na}_{2} \mathrm{SO}_{4}$. Dann wurde unter vermindertem Druck vom Lösungsmittel befreit und der feste Rückstand aus 2-Propanol umkristallisiert; Ausb. $39.8 \mathrm{~g} \mathrm{(71 \% )}$ farblose Kristalle, Schmp. $134^{\circ} \mathrm{C}$ (Subl.). - ${ }^{1} \mathrm{H}-\mathrm{NMR}: \delta=1.0-2.1\left(\mathrm{~m}, 17 \mathrm{H} ; \mathrm{C}_{6} \mathrm{H}_{11}, \mathrm{CCH}_{2} \mathrm{C}\right.$ von $\left.\mathrm{NC}_{5} \mathrm{H}_{10}\right), 2.5-2.6\left(\mathrm{~m}, 4 \mathrm{H} ; \mathrm{CCH}_{2} \mathrm{~N}\right.$ von $\left.\mathrm{NC}_{5} \mathrm{H}_{10}\right), 3.39\left(, \mathrm{sc}^{*}, 2 \mathrm{H}\right.$; $\mathrm{C} \equiv \mathrm{CCH}_{2} \mathrm{~N}$ ), 2.9 (breites symmetrisches Signal, nicht lagekonstant, $1 \mathrm{H} ; \mathrm{OH}), 7.2-7.6\left(\mathrm{~m}, 5 \mathrm{H} ; \mathrm{C}_{6} \mathrm{H}_{5}\right) .-{ }^{13} \mathrm{C}-\mathrm{NMR}: \delta=23.9,25.8(2 \mathrm{C})$, 26.2, 26.3, 26.4, 27.6 und $28.0\left(\mathrm{C}-2\right.$ bis $\mathrm{C}-6, \mathrm{C}_{6} \mathrm{H}_{11} ; \mathrm{C}-3$ bis $\mathrm{C}-5$, $\left.\mathrm{NC}_{5} \mathrm{H}_{10}\right), 47.9\left(\mathrm{C} \equiv \mathrm{CCH}_{2} \mathrm{~N}\right), 50.1\left(\mathrm{C}-1, \mathrm{C}_{6} \mathrm{H}_{11}\right), 53.1(2 \mathrm{C})(\mathrm{C}-2$ und $\left.\mathrm{C}-6, \mathrm{NC}_{5} \mathrm{H}_{10}\right), 76.7(\mathrm{COH}), 81.9$ und $87.1(\mathrm{C} \equiv \mathrm{C}), 126.3(2 \mathrm{C})(\mathrm{C}-2 /$ $\left.\mathrm{C}-6, \mathrm{C}_{6} \mathrm{H}_{5}\right), 127.4\left(\mathrm{C}-4, \mathrm{C}_{6} \mathrm{H}_{5}\right), 127.7$ (2C) $\left(\mathrm{C}-3 / \mathrm{C}-5, \mathrm{C}_{6} \mathrm{H}_{5}\right), 144.3$ $\left(\mathrm{C}-1, \mathrm{C}_{6} \mathrm{H}_{5}\right) .-$ FAB-MS: $m / z=312\left(100 \%,[\mathrm{M}+\mathrm{H}]^{+}\right)$.

$$
\begin{array}{lllll}
\mathrm{C}_{21} \mathrm{H}_{29} \mathrm{NO} \text { (311.5) } & \text { Ber. C } 80.98 \text { H } 9.38 \text { N } 4.50 \\
& \text { Gef. C } 80.6 \text { H } 9.5 \text { N } 4.7
\end{array}
$$

(R)-1-Cyclohexyl-1-phenyl-4-piperidino-2-butin-1-ol [(R)-2]:

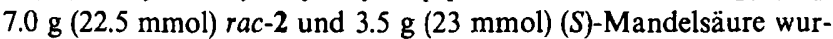
den in $150 \mathrm{ml}$ siedendem Acetonitril gelöst. Nach Filtration der heißen Lösung ließ man auf Raumtemp. abkühlen, versetzte mit einigen Impfkristallen (erhalten durch Spontankristallisation aus kleineren Analogansätzen) und lieB $24 \mathrm{~b}$ bei ca. $20^{\circ} \mathrm{C}$ ungestört stehen. Die gebildeten Mandelat-Kristalle (ca. $4.2 \mathrm{~g}$ ) wurden durch Filtration isoliert und durch dreimaliges Umkristallisieren aus Acetonitril (1 g Kristallisat pro $15 \mathrm{ml}$ heißes Acetonitril; Kristallisation bei ca. $20^{\circ} \mathrm{C}$ ) gereinigt. Das so erhaltene Mandelat (ca. $3.3 \mathrm{~g}$ ) wurde mit $200 \mathrm{ml} 0.1 \mathrm{M}$ Natronlauge versetzt und die dadurch freigesetzte Base durch Extraktion mit Diethylether isoliert. Die etherische Lösung wurde mehrmals mit etwas Wasser extrahiert, sodann mit wasserfreiem $\mathrm{Na}_{2} \mathrm{SO}_{4}$ getrocknet und unter vermindertem Druck vom Lösungsmittel befreit. Nach Trocknung des festen Rückstandes i. Vak. reinigte man diesen durch zweimaliges Umkristallisieren aus Aceton und erhielt $1.9 \mathrm{~g}$ [54\% Ausb., bezogen auf den Anteil von $(R)$ - 2 im eingesetzten Racemat rac-2] farblose Kristalle, Schmp. $126.5^{\circ} \mathrm{C},[\alpha]_{889}^{27}=+12\left(c=0.5, \mathrm{CHCl}_{3}\right)$, Enantiomerenreinheit ee $=99.7 \%$ [vgl. Kapitel b) des exp. Teils].

$$
\mathrm{C}_{21} \mathrm{H}_{29} \mathrm{NO} \text { (311.5) Ber. C } 80.98 \text { H } 9.38 \text { N } 4.50
$$$$
\text { Gef. C } 80.8 \text { H } 9.4 \text { N } 4.6
$$

(S)-1-Cyclohexyl-1-phenyl-4-piperidino-2-butin-1-ol [(S)-2]: Darstellung analog $\mathrm{zu}(R)-2$ durch Umsetzung von rac-2 mit $(R)$-Mandelsäure; Ausb. $51 \%$, Schmp. $126.5^{\circ} \mathrm{C},[\alpha]_{589}^{27}=-12(c=0.5$, $\mathrm{CHCl}_{3}$ ), Enantiomerenreinheit ee $=99.7 \%$.

$$
\begin{array}{llll}
\mathrm{C}_{21} \mathrm{H}_{29} \mathrm{NO} \text { (311.5) } & \text { Ber. C } 80.98 \text { H } 9.38 \text { N } 4.50 \\
& \text { Gef. C } 80.6 \text { H } 9.4 \text { N } 4.4
\end{array}
$$

(R)-1-Cyclohexyl-1-phenyl-4-piperidino-1-butanol-hydrochlorid $[(R)$-Hexahydro-Difenidol-hydrochlorid, $(R)-1 \mathbf{a} \cdot \mathrm{HCl}]$ : Eine Lösung von $2.0 \mathrm{~g}(6.4 \mathrm{mmol})(R)-2$ in $75 \mathrm{ml}$ Methanol wurde mit $75 \mathrm{mg}$ eines Pd/C-Katalysators (Pd-Anteil $10 \mathrm{Gew} . \%$ ) versetzt. Dann rührte man $2 \mathrm{~h}$ bei Raumtemp. im Wasserstoffstrom (vollständiger Umsatz, 'H-NMR-Kontrolle), filtrierte, befreite das Filtrat unter vermindertem Druck vom Lösungsmittel und versetzte den Rückstand mit $12.8 \mathrm{ml}$ einer $0.5 \mathrm{M}$ etherischen $\mathrm{HCl}$-Lösung $(6.4$ mmol $\mathrm{HCl}$ ). Der sich bildende Feststoff wurde durch Filtration isoliert und durch zweimaliges Umkristallisieren aus 2-Propanol/ Diethylether $(2: 1)$ gereinigt. Nach Trocknung i. Vak. erhielt man $1.6 \mathrm{~g} \mathrm{(71 \% )}$ farblose Kristalle, Schmp. $258^{\circ} \mathrm{C}$ (Subl. ab ca. $170^{\circ} \mathrm{C}$ ), $[\alpha]_{589}^{27}=-29\left(c=0.5, \mathrm{CHCl}_{3}\right)$, Enantiomerenreinheit ee $=99.7 \%$ (vgl. Kapitel b). - 'H-NMR: $\delta=1.0-2.3\left(\mathrm{~m}, 21 \mathrm{H} ; \mathrm{C}_{6} \mathrm{H}_{11}, \mathrm{CCH}_{2} \mathrm{C}\right.$ von $\left.\mathrm{C}\left[\mathrm{CH}_{2}\right]_{3} \mathrm{NC}_{5} \mathrm{H}_{10}\right), 2.4-2.6,2.8-3.05, \quad 3.25-3.35$ und
3.45-3.55 (m, 6H; $\left.\mathrm{CCH}_{2} \mathrm{~N}\right), 3.65$ (breites symmetrisches Signal, $1 \mathrm{H}$; $\mathrm{OH}), 7.15-7.35\left(\mathrm{~m}, 5 \mathrm{H} ; \mathrm{C}_{6} \mathrm{H}_{5}\right), 11.1$ (breites symmetrisches Signal; $\mathrm{NH}) .-{ }^{13} \mathrm{C}-\mathrm{NMR}: \delta=18.8,22.1,22.4(2 \mathrm{C}), 26.3,26.5,26.6,26.7$ und $27.4\left(\mathrm{C}-2\right.$ bis C-6, $\mathrm{C}_{6} \mathrm{H}_{11} ; \mathrm{C}-3$ bis C-5, $\mathrm{NC}_{5} \mathrm{H}_{10}, \mathrm{OCCH}_{2} \mathrm{CH}_{2}$ $\left.\mathrm{CH}_{2} \mathrm{~N}\right), 35.8\left(\mathrm{OCCH}_{2} \mathrm{CH}_{2} \mathrm{CH}_{2} \mathrm{~N}\right), 49.4\left(\mathrm{C}-1, \mathrm{C}_{6} \mathrm{H}_{11}\right), 52.9,53.3$ und $57.6\left(\mathrm{CCH}_{2} \mathrm{~N}\right), 78.9(\mathrm{COH}), 125.9(2 \mathrm{C}),\left(\mathrm{C}-2 / \mathrm{C}-6, \mathrm{C}_{6} \mathrm{H}_{5}\right), 126.3$ $\left(\mathrm{C}-4, \mathrm{C}_{6} \mathrm{H}_{5}\right), 127.9(2 \mathrm{C}),\left(\mathrm{C}-3 / \mathrm{C}-5, \mathrm{C}_{6} \mathrm{H}_{5}\right), 144.3\left(\mathrm{C}-1, \mathrm{C}_{6} \mathrm{H}_{5}\right)$. - FABMS: $m / z=316\left(100 \%\right.$, Kation), $98\left(35 \%, \mathrm{CH}_{2}=\mathrm{NC}_{5} \mathrm{H}_{10}^{+}\right)$.

$$
\begin{array}{llll}
\mathrm{C}_{21} \mathrm{H}_{34} \mathrm{ClNO}(352.0) & \text { Ber. C } 71.66 \text { H } 9.74 \text { N } 3.98 \\
& \text { Gef. C } 71.6 \text { H } 9.7 \text { N } 3.9
\end{array}
$$

(S)-1-Cyclohexyl-1-phenyl-4-piperidino-1-butanol-hydrochlorid [(S)-Hexahydro-Difenidol-hydrochlorid, $(S)-1 \mathbf{a} \cdot \mathrm{HCl}]$ : Analog zur Darstellung von $(R)-1 \mathrm{a} \cdot \mathrm{HCl}$. Ausb. $73 \%$, Schmp. $258^{\circ} \mathrm{C}$ (Subl. ab $\left.170^{\circ} \mathrm{C}\right),[\alpha]_{589}^{27}=+29\left(c=0.5, \mathrm{CHCl}_{3}\right)$, Enantiomerenreinheit ee $=$ 99.7\% (vgl. Kapitel b). Die spektroskopischen Daten des Produktes stimmten mit den für $(R)-1 \mathrm{a} \cdot \mathrm{HCl}$ angegebenen Werten überein.

$$
\mathrm{C}_{21} \mathrm{H}_{34} \mathrm{ClNO}(352.0) \quad \text { Ber. C } 71.66 \mathrm{H} 9.74 \mathrm{~N} 3.98
$$

Gef. C 71.7 H $9.8 \quad$ N 3.9

$\{1-[(S)-1-C y c l o h e x y l-1$-hydroxy-1-phenylbutyl]piperidinium $\}$. $(R)$-mandelat $\left[(S)-\mathbf{1 a} \cdot(R)-\mathrm{C}_{6} \mathrm{H}_{5} \mathrm{CH}(\mathrm{OH}) \mathrm{COOH}\right]$ : Durch Umsetzung von $75 \mathrm{mg}(0.21 \mathrm{mmol})(S)-1 \mathrm{a} \cdot \mathrm{HCl}$ mit $2.1 \mathrm{ml} 0.1 \mathrm{M}$ Natronlauge setzte man die entsprechende Base (S)-1 a frei, extrahierte diese mit $10 \mathrm{ml}$ Diethylether, trocknete mit wasserfreiem $\mathrm{Na}_{2} \mathrm{SO}_{4}$ und versetzte die etherische Lösung mit $38 \mathrm{mg}(0.25 \mathrm{mmol})(R)$-Mandelsäure. Der sich bildende Feststoff wurde durch Filtration isoliert und aus 2-Propanol/Diethylether (1:2) umkristallisiert; Ausb. $61 \mathrm{mg}(66 \%)$ farblose Kristalle.

\section{b) Kalorimetrische Bestimmung der Enantiomerenreinheiten}

Kalorimeter: FP 84 DSC/TOA, Fa. Mettler, Greifensee/Schweiz. Die Kalibrierung des Gerätes sowie die Durchführung und Auswertung der Messungen erfolgten wie in Lit. ${ }^{21,30)}$ beschrieben. Die Ergebnisse der kalorimetrischen Untersuchungen von $r a c-2,(R)-2$ und (S)-2 sind in Tab. 1 zusammengestellt. Die Ungenauigkeit der Enthalpiemessungen betrug $\pm 2.5 \%$, die der Temperaturmessung $\pm 0.2 \mathrm{~K}$ und die der Reinheitsbestimmung $\pm 50 \%$, bezogen auf die Verunreinigung. Das Phasendiagramm (Abb. 1) wurde aus den ermittelten Daten für die racemische Verbindung nach $\ln [4 X$ $(1-X)]=2 \Delta_{\text {fus }} H^{\ominus} / R\left(1 / T_{\text {fus }}-1 / T\right)$ und für die Enantiomere nach $\ln (X)=\Delta_{\text {fus }} H^{\ominus} / R\left(1 / T_{\text {fus }}-1 / T\right)$ berechnet ${ }^{31)}$. Während der Racematspaltung wurden die Anreicherungs-Fortschritte qualitativ aus den Schmelzpeaks der Gemische abgeschätzt.

\section{c) Röntgenstrukturanalyse ${ }^{321}$}

$(S)-1 \mathrm{a} \cdot(R)-\mathrm{C}_{6} \mathrm{H}_{5} \mathrm{CH}(\mathrm{OH}) \mathrm{COOH}$ kristallisiert aus 2-Propanol/ Diethylether $(1: 2)$ in der monoklinen Raumgruppe $P 2_{1}$ mit den aus 15 Reflexen $( \pm h k l)$ diffraktometrisch bestimmten Gitterkonstanten $a=608.3(1), b=2614.5(5), c=864.2(1) \mathrm{pm}, \beta=102.43(1)^{\circ}, Z=$ $2, d_{\text {ber }}=1.16 \mathrm{~g} / \mathrm{cm}^{3}$. Die Beugungsintensitäten wurden bei $293 \mathrm{~K}$ auf einem Syntex-Vierkreisdiffraktometer (Typ $P 2_{1}$ ) unter Verwendung von monochromatischer $\mathrm{Cu}-K_{x}$-Strahlung $(\lambda=154.18 \mathrm{pm})$ im $\Theta-2 \Theta$-Betrieb $\left(3^{\circ} \leq 2 \Theta \leq 135^{\circ}\right)$ gemessen. Die Meßgesch windigkeit variierte in Abhängigkeit von der Reflexintensität zwischen 2.93 und $29.30^{\circ} / \mathrm{min}$.

Bei der Datenreduktion wurden die Lorentz-, die Polarisationssowie eine empirische Absorptionskorrektur $\left(\mu=0.526 \mathrm{~mm}^{-1}\right)$ durchgeführt. Für die Verfeinerung wurden 3878 der gemessenen 4785 Reflexe mit $F \geq 4.0 \sigma(F)$ verwendet. Die Struktur wurde mit direkten Methoden und Differenz-Fourier-Synthesen gelöst. Die Wasserstoffatom-Positionen wurden mit Ausnahme des OH-Protons des Anions teilweise geometrisch berechnet, teilweise aus Differenz-Fourier-Synthese ermittelt und isotrop verfeinert. Die Verfej- 
nerung konvergierte bei $R=0.064$. Im letzten Verfeinerungszyklus war das Verhältnis von Parameteränderung zu geschätzter Standardabweichung für alle Parameter kleiner als $1 \%$. Eine abschlieBende Differenz-Fourier-Synthese ergab für die größten Elektronendichtemaxima Werte von $0.64 \times 10^{6} \mathrm{e} / \mathrm{pm}^{3}$. Neben eigenen Programmen wurde das Programm SHELX-76 ${ }^{33)}$ verwendet. Es wurden komplexe Atomformfaktoren ${ }^{34)}$ benutzt. - Die Atomkoordinaten sind in Tab. 3, ausgewählte Bindungsparameter in Tab. 4 mit dem in Abb. 2 verwendeten Numerierungsschema wiedergegeben.

Tab. 3. Lageparameter der Atome von $(\mathrm{S})-\mathbf{1} \mathbf{a} \cdot(\mathrm{R})-\mathrm{C}_{6} \mathrm{H}_{5} \mathrm{CH}(\mathrm{OH}) \mathrm{COOH}$

\begin{tabular}{lcrrr}
\hline \multicolumn{7}{c}{ X/A } & Y/B & Z/C & UEO \\
\hline$C(1)$ & $-0.4209(5)$ & $0.1419(0)$ & $-0.7212(4)$ & $0.063(1)$ \\
$O(1)$ & $-0.6118(4)$ & $0.1245(1)$ & $-0.6656(3)$ & $0.086(1)$ \\
$C(111)$ & $-0.3384(5)$ & $0.1901(1)$ & $-0.6263(4)$ & $0.060(1)$ \\
$C(112)$ & $-0.1437(6)$ & $0.2142(1)$ & $-0.6450(5)$ & $0.074(1)$ \\
$C(113)$ & $-0.0703(8)$ & $0.2587(2)$ & $-0.5608(6)$ & $0.087(1)$ \\
$C(114)$ & $-0.1868(9)$ & $0.2787(2)$ & $-0.4551(6)$ & $0.094(1)$ \\
$C(115)$ & $-0.3819(9)$ & $0.2552(2)$ & $-0.4375(5)$ & $0.094(1)$ \\
$C(116)$ & $-0.4568(7)$ & $0.2107(2)$ & $-0.5211(5)$ & $0.079(1)$ \\
$C(121)$ & $-0.4852(6)$ & $0.1567(1)$ & $-0.8998(4)$ & $0.067(1)$ \\
$C(122)$ & $-0.6659(8)$ & $0.1993(2)$ & $-0.9339(5)$ & $0.095(1)$ \\
$C(123)$ & $-0.7236(10)$ & $0.2148(2)$ & $-1.1072(6)$ & $0.110(1)$ \\
$C(124)$ & $-0.7898(9)$ & $0.1703(2)$ & $-1.2110(5)$ & $0.097(1)$ \\
$C(125)$ & $-0.6125(9)$ & $0.1290(2)$ & $-1.1831(5)$ & $0.096(1)$ \\
$C(126)$ & $-0.5567(7)$ & $0.1124(2)$ & $-1.0094(5)$ & $0.082(1)$ \\
$C(2)$ & $-0.2362(6)$ & $0.1009(1)$ & $-0.6920(5)$ & $0.073(1)$ \\
$C(3)$ & $-0.1367(12)$ & $0.0872(2)$ & $-0.5194(6)$ & $0.132(2)$ \\
$C(4)$ & $-0.2013(18)$ & $0.0515(2)$ & $-0.4391(6)$ & $0.181(3)$ \\
N(4) & $-0.2610(5)$ & $-0.0024(1)$ & $-0.4943(3)$ & $0.071(1)$ \\
$C(5)$ & $-0.0650(6)$ & $-0.0360(2)$ & $-0.4836(5)$ & $0.084(1)$ \\
$C(6)$ & $-0.1309(8)$ & $-0.0885(2)$ & $-0.5323(7)$ & $0.107(2)$ \\
$C(7)$ & $-0.2895(9)$ & $-0.1109(2)$ & $-0.4349(8)$ & $0.114(2)$ \\
$C(8)$ & $-0.4869(7)$ & $-0.0767(2)$ & $-0.4484(6)$ & $0.106(1)$ \\
$C(9)$ & $-0.4139(8)$ & $-0.0238(2)$ & $-0.3983(5)$ & $0.098(1)$ \\
$C(10)$ & $-0.8044(6)$ & $-0.0165(1)$ & $-0.9998(4)$ & $0.068(1)$ \\
$O(10)$ & $-1.0085(5)$ & $0.0101(1)$ & $-1.0339(3)$ & $0.098(1)$ \\
$C(101)$ & $-0.8376(5)$ & $-0.0742(1)$ & $-1.0096(3)$ & $0.064(1)$ \\
$C(102)$ & $-0.6824(7)$ & $-0.1054(2)$ & $-1.0582(5)$ & $0.091(1)$ \\
$C(103)$ & $-0.7164(10)$ & $-0.1582(2)$ & $-1.0688(7)$ & $0.116(2)$ \\
$C(104)$ & $-0.8971(11)$ & $-0.1787(2)$ & $-1.0233(7)$ & $0.113(2)$ \\
$C(105)$ & $-1.0462(9)$ & $-0.1491(2)$ & $-0.9697(7)$ & $0.105(1)$ \\
$C(106)$ & $-1.0191(7)$ & $-0.0957(2)$ & $-0.9645(5)$ & $0.083(1)$ \\
$C(11)$ & $-0.6668(6)$ & $-0.0022(1)$ & $-0.8352(4)$ & $0.063(1)$ \\
$O(11)$ & $-0.7662(4)$ & $0.0234(1)$ & $-0.7510(3)$ & $0.078(1)$ \\
$O(12)$ & $-0.4679(4)$ & $-0.0161(1)$ & $-0.8015(3)$ & $0.075(1)$ \\
\hline & & & &
\end{tabular}

Tab. 4. Ausgewählte Bindungslängen ${ }^{\text {a) }}[\mathrm{pm}]$ und -winkel $\left[{ }^{\circ}\right]$ von $(S)-1 \mathbf{a} \cdot(R)-\mathrm{C}_{6} \mathrm{H}_{5} \mathrm{CH}(\mathrm{OH}) \mathrm{COOH}$

\begin{tabular}{|c|c|c|c|c|c|c|c|}
\hline$O(1)$ & $-c(1)$ & 142.31 & 4) & $c(111)$ & $-C(1)$ & 152.7( & 4) \\
\hline$c(121)$ & $-c(1)$ & 155.7 ( & 5) & $c(2)$ & $-c(1)$ & $153.5 i$ & 4) \\
\hline$C(122)$ & $-C(121)$ & 154.8 ( & 6) & $C(126)$ & $-c(121)$ & 150.1 & 5) \\
\hline$c(123)$ & $-c(122)$ & 151.71 & 6) & $C(124)$ & $-c(123)$ & 147.1 ( & 7) \\
\hline$c(125)$ & $-C(124)$ & 150.81 & 7) & $c(126)$ & $-c(125)$ & $152.9 i$ & 6) \\
\hline$c(3)$ & $-c(2)$ & 152.6 ( & 6) & $c(4)$ & $-c(3)$ & 127.4 ( & 9) \\
\hline$N(4)$ & $-C(4)$ & 150.7 ( & 6) & $c(5)$ & $-N(4)$ & 146.81 & 5) \\
\hline C(9) & $-N(4)$ & 148.3 ( & 6) & $c(6)$ & $-c(5)$ & 146.51 & 7) \\
\hline$c(7)$ & $-C(6)$ & 152.7 ( & 9) & $c(8)$ & $-c(7)$ & 148.11 & 7) \\
\hline c(9) & $-c(8)$ & 148.9 & 8) & $O(10)$ & $-c(10)$ & 139.8( & 4) \\
\hline$c(101)$ & $-c(10)$ & 152.1 ( & 5) & $c(11)$ & $-c(10)$ & 153.41 & 4) \\
\hline$O(11)$ & $-c(11)$ & 123.71 & 5) & $O(12)$ & $-c(11)$ & $123.7 i$ & 4) \\
\hline$H(1)$ & $-\infty(1)$ & 85 & 3) & $\mathrm{H}(4)$ & $-N(4)$ & 92( & 3) \\
\hline$c(111)$ & $-c(1)$ & $-(1)$ & $106.3(3)$ & $C(121)$ & $-C(1)$ & $-\infty(1)$ & $111.4(3)$ \\
\hline$c(121)$ & $-c(1)$ & $-C$ (11i) & $108.1(2)$ & $c(2)$ & $-c(1)$ & $-0(1)$ & $210.0(2)$ \\
\hline$c(2)$ & $-c(1)$ & $-c(111)$ & 110.1 ( 3$)$ & $c(2)$ & $-c(1)$ & $-c(121)$ & $110.8(3)$ \\
\hline$c(122)$ & $-C(121)$ & $-c(1)$ & $112.6(3)$ & $c(126)$ & ) $-c(121)$ & $-c(1)$ & $114.2(3)$ \\
\hline$c(126)$ & $-c(121)$ & $-c(122)$ & $109.8(3)$ & $c(123)$ & $j-c(122)$ & $-c(121)$ & $112.5(4)$ \\
\hline$c(124)$ & $-C(123)$ & $-c(122)$ & $111.5(4)$ & $c(125)$ & $-c(124)$ & $-c(123)$ & $112.0(4)$ \\
\hline$C(126)$ & $-c(125)$ & $-c(124)$ & $111.2(4)$ & $c(125)$ & $-c(126)$ & $j-c(121)$ & $111.9(3)$ \\
\hline$c(3)$ & $-c(2)$ & $-c(1)$ & $116.5(4)$ & $c(4)$ & $-c(3)$ & $-c(2)$ & $126.8(5)$ \\
\hline N(4) & $-c(4)$ & $-c(3)$ & 126.5 ( 5) & $c(5)$ & $-\mathrm{N}(4)$ & $-C(4)$ & 113.7 (5) \\
\hline c(9) & $-N(4)$ & $-c(4)$ & $108.3(4)$ & $c(9)$ & $-N(4)$ & $-c(5)$ & $109.7(3)$ \\
\hline$c(6)$ & $-c(5)$ & $-N(4)$ & $111.9(3)$ & $c(7)$ & $-c(6)$ & $-c(5)$ & $111.6(5)$ \\
\hline$C(8)$ & $-c(7)$ & $-c(6)$ & $108.8(4)$ & $c(9)$ & $-c(8)$ & $-c(7)$ & $110.6(4)$ \\
\hline$c(8)$ & $-c(9)$ & $-N(4)$ & $111.8(4)$ & $c(101)$ & $-c(10)$ & $-O(10)$ & $112.3(3)$ \\
\hline$c(11)$ & $-c(10)$ & $-O(10)$ & $110.9(3)$ & $c(11)$ & $-c(10)$ & $-C(101)$ & $109.5(2)$ \\
\hline$O(11)$ & $-c(11)$ & $-c(10)$ & 116.1 (3) & $O(12)$ & $-c(11)$ & $-c(10)$ & $117.2(3)$ \\
\hline$O(12)$ & $-c(11)$ & $-\infty(11)$ & $126.7(3)$ & & & & \\
\hline
\end{tabular}

\section{d) Pharmakologische Untersuchungen}

Die Prüfungen auf antimuscarinische Aktivität erfolgten am isolierten, elektrisch gereizten linken Atrium und an der isolierten Longitudinalmuskulatur des Ileums vom Meerschweinchen bei einer Temperatur von $32^{\circ} \mathrm{C}$ und einem $\mathrm{pH}$-Wert von 7.4. Als Agonist wurde Arecaidinpropargylester ${ }^{2)}$ verwendet. Die Stimulation der Vorhöfe erfolgte mit Rechteckimpulsen von einer Dauer von $3 \mathrm{~ms}$ bei $2 \mathrm{~Hz}$ und 5-10 V. Die Registrierung der Effekte an den Organpräparaten wurde mit Hilfe eines elektromechanischen Kraftumwandlers (Ileum: isotonisch; Atrium: isometrisch) vorgenommen. Die Auswertung der Versuchsergebnisse erfolgte nach Arunlakshana und Schild ${ }^{27-29}$. Die einzelnen Antagonisten wurden in steigenden Konzentrationen (log-Abstand $=0.48$; mindestens drei Konzentrationen; $N=3-5$ ) getestet. Zur Charakterisierung der Affinitäten der Enantiomere von 1a und 2 zu den atrialen $M 2 \alpha$ und den ilealen M2 $\beta$-Rezeptoren wurden die $\mathrm{p} A_{2}$-Werte bzw. die Dissoziationskonstanten $K_{\mathrm{D}} \quad\left(\mathrm{p} A_{2}=-\log K_{\mathrm{D}}\right)$ herangezogen (Tab. 2, Abb. 3). Die Steigungen in den Arunlakshana-Schild-Darstellungen dienten als wichtiges Kriterium für einen kompetitiven Antagonismus. Alle pharmakologischen Parameter wurden als Mittelwerte \pm Standardfehler des Mittelwertes ausgedrückt. Differenzen von Mittelwerten wurden als signifikant angesehen, wenn $p<$ 0.05 war $\left(t\right.$-Test). - Für weitere experimentelle Details siehe Lit. ${ }^{25)}$.

\section{CAS-Registry-Nummern}

(R)-1 a: $117828-66-7 /(S)-1$ a: $117828-64-5 /(R)-1$ a $\cdot \mathrm{HCl}: 117828-$ 62-3/(S)-1 a $\cdot \mathrm{HCl}: 117828-63-4 /(S)-1 \mathrm{a} \cdot(R)-\mathrm{PhCH}(\mathrm{OH}) \mathrm{CO}_{2} \mathrm{H}$ : 117828-65-6 / (R)-2: 117894-35-6 / (S)-2: 117894-36-7 / ( \pm -2: 117828-61-2 / 3-Piperidino-1-propin: 5799-75-7 / Cyclohexylphenylketon: $712-50-5$

${ }^{1)}$ R. Tacke, H. Linoh, H. Zilch, J. Wess, U. Moser, E. Mutschler, G. Lambrecht, Liebigs Ann. Chem. 1985, 2223.

2) E. Mutschler, G. Lambrecht, Trends Pharmacol. Sci. (Suppl.) 5 (1984) 39.

${ }^{3)}$ G. Lambrecht, E. Mutschler, U. Moser, J. Riotte, M. Wagner, J. Wess, G. Gmelin, R. Tacke, H. Zilch in International Symposium on Muscarinic Cholinergic Mechanisms (S. Cohen, M. Sokolovsky, Ed.), S. 245 - 253, Freund Publishing House Ltd., London 1987.

4) E. Mutschler, G. Gmelin, U. Moser, J. Wess, G. Lambrecht in Pharmacology (M. J. Rand, C. Raper, Ed.), S. 67-75, Elsevier Science Publishers B. V., Amsterdam 1987.

5) G. Lambrecht, U. Moser, M. Wagner, J. Wess, G. Gmelin, K. Raseiner, C. Strohmann, R. Tacke, E. Mutschler, Trends Pharmacol. Sci. (Suppl.) 9 (1988) 82.

6) E. Mutschler, U. Moser, J. Wess, G. Lambrecht in Recent Advances in Receptor Chemistry (C. Melchiorre, M. Giannella, Ed.), S. 195-217, Elsevier Science Publishers B. V., Amsterdam 1988.

7) M. Herawi, G. Lambrecht, E. Mutschler, U. Moser, A. Pfeiffer, Gastroenterology 94 (1988) 630

${ }^{8)}$ M. Waelbroeck, M. Tastenoy, J. Camus, J. Christophe, C. Strohmann, H. Zilch, R. Tacke, E. Mutschler, G. Lambrecht, Br. J. Pharmacol., eingereicht zur Publikation.

9) M. Eltze, G. Gmelin, J. Wess, C. Strohmann, R. Tacke, E. Mutschler, G. Lambrecht, Eur. J. Pharmacol., im Druck.

10) H. Fuder, H. Kilbinger, H. Müller, Eur. J. Pharmacol. 113 (1985) 125.

${ }^{11}$ R. M. Eglen, R. L. Whiting, J. Auton. Pharmacol. 5 (1986) 323.

12) N. J. M. Birdsall, E. C. Hulme, M. Keen, E. K. Pedder, D. Poyner, J. M. Stockton, M. Wheatley, Biochem. Soc. Symp. 52 (1986) 23.

13) S. Batra, A. Björklund, H. Hedlund, K.-E. Andersson, J. Auton. Nervous System 20 (1987) 129.

14) E. E. Daniel, J. Jury, P. Bowker, Can. J. Physiol. Pharmacol. 65 (1987) 1903.

15) B. Baudiere, E. Monferini, E. Giraldo, H. Ladinsky, J. P. Bali, Biochem. Pharmacol. 36 (1987) 2957.

${ }^{16)}$ M. Waelbroeck, J. Camus, J. Winand, J. Christophe, Life Sci. 41 (1987) 2235 
${ }^{17}$ M. Eltze, Eur. J. Pharmacol., 151 (1988) 205.

18) W. S. Sheldrick, H. Linoh, R. Tacke, G. Lambrecht, U. Moser, E. Mutschler, J. Chem. Soc., Dalton Trans. 1985, 1743.

i9) R. Tacke, H. Linoh, D. Schomburg, L. Ernst, U. Moser, E. Mutschler, G. Lambrecht, Liebigs Ann. Chem. 1986, 242.

${ }^{20)} \mathrm{G}$. Lambrecht, E. Mutschler in Innovative Approaches in Drug Research (A. F. Harms, Ed.), S. 353-370, Elsevier Science Publishers B. V., Amsterdam 1986.

21) R. Tacke, H. Linoh, L. Ernst, U. Moser, E. Mutschler, S. Sarge, H. K. Cammenga, G. Lambrecht, Chem. Ber. 120 (1987) 1229.

22) Vorläufige Mitteilung über Teilergebnisse: R. Feifel, U. Moser, A. Aasen, C. Strohmann, R. Tacke, E. Mutschler, G. Lambrecht, Xth International Symposium on Medicinal Chemistry, Budapest 1988, Abstracts, S. 233.

23) $\mathrm{rac}-2$ ist bereits als Hydrochlorid beschrieben worden, wurde jedoch bisher auf anderem Wege dargestellt: R. Dahlbom, B. Karlen, S. Rämsby, I. Kraft, R. Mollberg, Acta Pharm. Suecica 1 (1964) 237.

24) D. Rogers, Acta, Cryst., Sect. A, 37 (1981) 734.
25) C. Melchiorre, P. Angeli, G. Lambrecht, E. Mutschler, M. T. Picchio, J. Wess, Eur. J. Pharmacol. 144 (1987) 117.

26) R. M. Eglen, R. L. Whiting, Br. J. Pharmacol. 90 (1987) 701

27) D. McKay, J. Pharm. Pharmacol. 30 (1978) 312

28) R. J. Tallarida, A. Cowan, M. W. Adler, Life Sci. 25 (1979) 637.

29) O. Arunlakshana, H. O. Schild, Br. J. Pharmacol. 14 (1959) 48.

30) S. Sarge, H. K. Cammenga, Thermochim. Acta 94 (1985) 17.

${ }^{31)} \mathrm{G}$. Kortüm, H. Lachmann, Einführung in die chemische Thermodynamik, 7. Aufl., S. 288-298, Verlag Chemie, Weinheim 1981.

32) Weitere Einzelheiten zur Kristallstrukturbestimmung können beim Fachinformationszentrum Energie Physik Mathematik, D-7514 Eggenstein-Leopoldshafen 2, unter Angabe der Hinterlegungsnummer CSD-53497, der Autoren und des Zeitschriftenzitats angefordert werden.

33) G. M. Sheldrick, unveröffentlichte Ergebnisse.

${ }^{34)}$ D. T. Cromer, J. T. Waber in International Tables for Crystallography (J. A. Ibers, W. C. Hamilton, Ed.), Bd. IV, S. 99-102 und S. 149, Kynoch Press, Birmingham 1974.

$[169 / 88]$ 